ich zudem das Thema Lehrerbildung auf unterschiedlichen Ebenen, seit $200 \mathrm{I}$ als Herausgeberin der Buchreihe „Mathematics Teacher Education and Development“ bei Springer sowie als Koordinatorin diesbezüglicher Topic Groups auf internationalen Tagungen, als Wissenschaftlerin im Rahmen eigener Forschungsprojekte - aktuell in einem interdisziplinären Drittmittelprojekt zur Entwicklung (mathematik-)diagnostischer Kompetenzen im Studium - und auch hochschulpolitisch wie wissenschaftlich als stellvertretende Direktorin der Bielefeld School of Education.
Aus dieser Perspektive verfolge ich auch seit einigen Jahren mit großem Interesse die Beteiligung der DMV an Diskussionen und Papieren zur Lehrerausbildung. Ich würde mich gern in der DMV engagieren und mich dort besonders für die Aus-, Fort- und Weiterbildung von Mathematiklehrer/-innen einsetzen - nicht nur aber auch angesichts der aktuellen Herausforderungen im Kontext von Inklusion und der Integration geflüchteter Kinder und Jugendlicher in unsere Schulen.

\title{
DMV-Ansprechpartner/innen vor Ort
}

- Aachen: Gabriele Nebe - Augsburg: Jost-Hinrich Eschenburg - Augsburg: Ralf Werner - Bamberg: Anna Susanne Steinweg - Bayreuth: Thomas Peternell a FU Berlin: Ehrhard Behrends, Günter M. Ziegler - TU Berlin: Michael Joswig, Martin Skutella - HU Berlin: Jürg Kramer - WIAS Berlin: Wolfgang König - FH Bielefeld: Claudia Cottin - U Bielefeld: Michael Röckner - Bochum: Peter Eichelsbacher, Thomas Skill - Bonn: Werner Ballmann — Braunschweig: Volker Bach —U Bremen: Dmitry Feichtner-Kozlov a JU Bremen: Dierk Schleicher - Chemnitz: Christoph Helmberg - Cottbus: Friedrich Sauvigny - Darmstadt: Stefan Ulbrich — Dresden: Andreas Thom Duisburg: Rüdiger Schultz - Düsseldorf: Kai Köhler — Erlangen: Günter Leugering $n$ Essen: Hans Niels Jahnke n Flensburg: Heinrich Lorenzen - Frankfurt am Main: Thorsten Theobald a TU Freiberg: Michael Eiermann - Freiburg: Sebastian Goette a Gießen: Thomas Bartsch - Göttingen: Thomas Schick — Greifswald: Michael Schürmann - Hagen: Winfried Hochstättler - Halle: Gernot Stroth - Hamburg: Benedikt Löwe - Hamburg-Harburg: Wolfgang Mackens - Hannover: Christine Bessenrodt - Heidelberg: Gebhard Böckle — Hildesheim: Jürgen Sander $\square$ Ho- henheim: Georg Zimmermann $\square$ Ilmenau: Carsten Trunk $\square$ Jena: Tobias Oertel-Jäger - Karlsruhe: Michael Plum — Kassel: Wolfram Koepf — Köln: Peter Littelmann — Konstanz: Claus Scheiderer - Leipzig: Hans-Bert Rademacher v Lübeck: Jürgen Prestin — Lüneburg: Silke Ruwisch — Magdeburg: Volker Kaibel - Mainz: Thorsten Raasch @ Mannheim: Alexander Schied n Marburg: Volkmar Welker — LMU München: Helmut Schwichtenberg — UniBW München: Cornelius Greither — TU München: Peter Gritzmann — Münster: Michael Joachim — HS Neubrandenburg: Gerd Teschke - Oldenburg: Daniel Grieser - Osnabrück: Holger Brenner - Paderborn: Torsten Wedhorn - Passau: Brigitte Forster-Heinlein - Passau: Niels Schwartz - Potsdam: Christian Bär n Regensburg: Guido Kings n Rostock: Roger Labahn ॥ Saarbrücken: Jörg Eschmeier - Siegen: NilsPeter Skoruppa - HfT Stuttgart: Peter Hauber —U Stuttgart: Timo Weidl - Trier: Jochen Wengenroth - Tübingen: Jürgen Hausen - HS Ulm: Günter Gramlich — Vechta: Martin Winter — Weimar: Klaus Gürlebeck n Wuppertal: Jens Hornbostel - Würzburg: Stefan Waldmann

DEUTSCHE MATHEMATIKER-VEREINIGUNG — VORSTAND UND PRÄSIDIUM Präsident Prof. Dr. Volker Bach, Institut für Analysis und Algebra, Technische Universität Braunschweig, Pockelsstraße 14, 38I06 Braunschweig, Tel. +49.53I 391 7429, vbach@tu-bs.de Vizepräsident Prof. Dr. Michael Röckner, Fakultät für Mathematik, Universität Bielefeld, Universitätsstraße 25, 33615 Bielefeld, Tel. +49.52I 106-4774 roeckner@math. uni-bielefeld.de Schatzmeister Prof. Dr. Etienne Emmrich, Institut für Mathematik, MA 5-3, Technische Universität Berlin, Straße des I7. Juni 136, 10623 Berlin, Tel. +49.3031425745, emmrich@math.tu-berlin.de Schriftführer Prof. Dr. Daniel Grieser, Universität Oldenburg, Institut für Mathathematik, Carl-von-Ossietzky-Straße 9-II, 26I29 Oldenburg, Tel. +49.44I.7983230 daniel.grieser@uni-oldenburg.de Herausgeber der Mitteilungen Prof. Dr. Michael Joswig (verantwortl.), TU Berlin, MA 6-2, Straße des 17. Juni 136, I0623 Berlin, Tel. +49.30.31475904 joswig@math.tu-berlin.de Weitere Präsidiumsmitglieder . Prof. Dr. Rudolf Grübel, Hannover , Prof. Dr. Hans-Christoph Grunau, Magdeburg (Herausgeber des Jahresberichtes der DMV) - Prof. Dr. Moritz Kaßmann, Bielefeld . Prof. Dr. Wolfram Koepf, Kassel (Verantwortlicher für www.mathematik.de) , Matthias Lippert (Lehrervertreter) - Prof. Dr. Bettina Rösken-Winter, Bochum . Prof. Dr. Katrin Wendland, Freiburg - Prof. Dr. Günter M. Ziegler, FU Berlin (Leiter Medien- und Netzwerkbüro der DMV) — Mitgliedsbeitrag 2016 (inkl. Bezug der Mitteilungen und einer gewählten Zeitschrift, Ausnahme: Studierende und Schüler beziehen nur die Mitteilungen) - regulär EUR 105,00 . bis zur Vollendung des 30. Lebensjahres EUR 50,00 . ermäßigt für Ehepaare und eingetragene Lebenspartnerschaften EUR 150,00 * ermäßigt für Studierende (Bachelor/Master/Diplom) und Schülerinnen und Schüler EUR 20,00 - Sonderbeitrag auf Antrag (z. B. bei Arbeitslosigkeit) EUR 20,00 . ermäßigt für Mitglieder der DPG/GI/GOR/GDM/MNU oder MUED EUR 90,00 EUR - ermäßigt für Reziprozitätsmitglieder (im Ausland wohnend und Vollmitglied einer Mathematischen Gesellschaft, mit der die DMV ein Reziprozitätsabkommen hat) EUR 70,00 : ermäßigt für Senioren EUR 70,00 - Zeitschriften (Jahresabo 2016 jeweils EUR 26,00), eine der folgenden Zeitschriften ist im Mitgliedsbeitrag enthalten: - Jahresbericht der DMV (Springer Verlag Heidelberg, 4 Hefte jährlich) - Journal für Mathematik-Didaktik (Springer Verlag Heidelberg, 2 Hefte jährlich) . Mathematische Semesterberichte (Springer Verlag Heidelberg, 2 Hefte jährlich) — DMV-Server dmv.mathematik.de www.mathematik.de - DOCUMENTA MATHEMATICA www.mathematik.uni-bielefeld.de/documenta/ n Medienbüro der DMV Thomas Vogt, FU Berlin (dmv.mathematik.de) - Netzwerkbüro der DMV Stephanie Schiemann, FU Berlin (dmv.mathematik.de) . Geschäftsstelle der DMV Geschäftsführerin Roswitha Jahnke (dmv.mathematik.de) • Bankverbindung Volksbank Freiburg 6955002 (BLZ 68090000 ), IBAN: DE66 6809 00000006955002 , BIC: GENODE6IFRI

Die Deutsche Mathematiker-Vereinigung e.V. ist durch den Körperschaftssteuerbescheid 2014 des Finanzamtes für Körperschaften Berlin I (Steuer-Nr. 27/640/5 I05I) vom 18.2. 2016, wegen „,Förderung von Wissenschaft und Forschung“ als wissenschaftlichen Zwecken dienend und zu den in $§ 5$ Absatz I Nr. 9 KStG bezeichneten Körperschaften gehörig anerkannt worden. Vereinseintrag: VR 380040 beim Amtsgericht Stuttgart. Umsatzsteuer-Identifikationsnummer: DE 165534138 